
\title{
Research S Surate \\ Metabolomic Spectra For Phenotypic Prediction of Malting Quality In Spring Barley
}

\section{Xiangyu Guo ( $\nabla$ Xiangyu.Guo@qgg.au.dk)}

Aarhus University

Ahmed Jahoor

Nordic Seed A/S

Just Jensen

Aarhus University

Pernille Sarup

Nordic Seed A/S

\section{Research Article}

Keywords: barley, malting quality, metabolomic prediction, MBLUP, PLSR, training population size

Posted Date: December 3rd, 2021

DOI: https://doi.org/10.21203/rs.3.rs-1113863/v1

License: (c) (i) This work is licensed under a Creative Commons Attribution 4.0 International License. Read Full License 


\section{$1 \quad$ Metabolomic Spectra For Phenotypic Prediction Of Malting}

\section{Quality In Spring Barley}

3 Xiangyu Guo ${ }^{1,2 *}$, Ahmed Jahoor ${ }^{3,4}$, Just Jensen ${ }^{1}$, Pernille Sarup ${ }^{3}$

$4{ }^{1}$ Center for Quantitative Genetics and Genomics, Aarhus University, 8830 Tjele, Denmark

$5 \quad{ }^{2}$ SEGES, Pig Research Centre, 8200, Aarhus, Denmark

$6 \quad{ }^{3}$ Nordic Seed A/S, 8300 Odder, Denmark

$7{ }^{4}$ Department of Plant Breeding, The Swedish University of Agricultural Sciences, 2353 Alnarp,

8 Sweden

$9{ }^{*}$ Correspondance:

10 Dr. Xiangyu Guo

11 Xiangyu.Guo@qgg.au.dk 


\section{Abstract}

13 The objectives were to investigate prediction of malting quality (MQ) phenotypes in different 14 locations using information from metabolomic spectra, and compare the prediction ability 15 using different models and different sizes of training population (TP).

16 A total of 2,667 plots of 564 malting spring barley lines from three years and two locations 17 were included. Five MQ traits were measured in wort produced from each individual plot. 18 Metabolomic features (MFs) used were 24,018 NMR intensities measured on each wort sample.

19 Models involved in the statistical analyses were a metabolomic best linear unbiased prediction

20 (MBLUP) model and a partial least squares regression (PLSR) model. Predictive ability within

21 location and across locations were compared using cross-validation methods.

22 The proportion of variance in MQ traits that could be explained by effects of MFs was above 230.9 for all traits. The prediction accuracy increased with increasing TP size but when the TP 24 size reached 1,000, the rate of increase was negligible. The number of components considered in the PLSR models can affect the performance of PLSR models and 20 components were optimal. The accuracy of individual plots and line means using leave-one-line-out crossvalidation ranged from 0.722 to 0.865 and using leave-one-location-out cross-validation ranged from 0.517 to 0.817 .

29 In conclusion, it is possible to carry out metabolomic prediction of MQ traits using MFs, the prediction accuracy is high and MBLUP is better than PLSR if the training population is larger than 100. The results have significant implications for practical barley breeding for malting quality.

\section{Keywords}

34 barley, malting quality, metabolomic prediction, MBLUP, PLSR, training population size 
Introduction

36 Brewing for alcohol production is the major end use of malt, and barley is the primary cereal used in production of malt because of optimal content of carbohydrates, dietary fibers, protein, vitamins and minerals ${ }^{1}$. In the process of brewing, the cereal needs to be malted. Under specific controlled conditions, cereal grains are sprouted and the young seedlings are grown for four to

40 six days in order to produce malt ${ }^{2}$. This process ensure a physical and biochemical transformation within the grain that is defined as malting ${ }^{1}$. During the process of malting, the cell-wall is degraded and protein is dissolved by hydrolytic enzymes such that the physical and the biochemical structure of the barley grain is modified in order to allow malt to be used in the subsequent stages of brewing ${ }^{3}$.

The quality of malt can directly affect the quality and quantity of brewed beer, thus malting quality (MQ) traits are important traits in breeding of barley to be used for malting. A series of MQ traits are defined in the malting industry. These include traits as extract yield and grain protein; alpha-amylase, beta-glucanase, beta-glucan, soluble protein, and free amino nitrogen in wort; and some physical properties such as diastatic power, viscosity, taste, flavor, haze and foam head retention ${ }^{4}$. Measurement of MQ traits is expensive and labor-intensive and the MQ traits have been demonstrated to have complex inheritance ${ }^{5,6}$. A detailed analysis of genetic variation in MQ traits in spring barley was provided by a previous study ${ }^{6}$, where a population of 1,329 spring barley lines from four breeding cycles were investigated and medium to high narrow sense heritabilities were found for the MQ traits included in this study. The organic compounds in a plant are mostly produced by the plant itself so that the photosynthetic and metabolic capacity of a plant is the primary factor determining its growth potential ${ }^{7}$. Metabolites are typical intermediates of biochemical reactions during the growth and development at all stages of plant life ${ }^{8}$. A comprehensive view of cellular metabolites can be provided by metabolomics, which is an approach to quantify the endogenous metabolites in 
60 cells and organisms. The development of metabolomics has contributed to the molecular and

61 biological characterization of various organisms. Especially in the area of crops, compared

62 with animals and microorganisms, metabolomics is of great importance since the crops produce

63 very large array of metabolites collectively ${ }^{9}$. Omics technologies like genomics,

64 transcriptomics, and metabolomics can be used in the investigation for the biological

65 background in different organisms ${ }^{10}$.

66 Nuclear magnetic resonance (NMR) spectroscopy is one of the technologies used to analyze

67 many metabolites simultaneously ${ }^{11}$. NMR can produce signal intensities, which can be treated

68 as an indicator of metabolites in a biological sample, were defined as metabolomic features

69 (MFs) ${ }^{12}$. A total of 24,018 MFs from barley wort were investigated in a previous study where

70 the genetic variation in the MFs was investigated using a univariate model and 8,604 MFs were

71 found to be significantly heritable ${ }^{13}$.

72 Partial least squares (PLS) was first developed for the modelling of information-scarce

73 situations in social sciences by Wold ${ }^{14}$. It is a latent variable approach which has been used to

74 find fundamental relationships between two matrices by modelling the inner covariance

75 structures ${ }^{15}$. PLS regression (PLSR) is the simplest PLS approach, and is a dimension

76 reduction method which has been widely used in chemometrics ${ }^{16}$. Similar with traditional

77 regression, PLSR relates two matrices by a linear multivariate model, but compared with

78 traditional regression, the structure of two matrices can also be modelled when using PLSR ${ }^{16}$.

79 The use of PLSR in chemistry first started in 1980s and has increased steadily for about 40

80 years, due to its appealing mathematical properties ${ }^{17}$. PLSR is able to analyze data sets with a

81 large number of explanatory variables compared to the number of observations, in cases of

82 noisy data, multi-collinearity, and incomplete variables in both the matrix of dependent

83 variables and the matrix of predictor variables ${ }^{16,18}$. 
84 Best linear unbiased prediction (BLUP), which is a method allowing prediction of random effects in a mixed model, was originally developed in animal breeding for prediction of breeding values (BVs) (Henderson, 1975). In the area of animal breeding, the selection of animals with highest BV was usually based on predicted/expected BVs (EBVs) derived from the records on the animals themselves and their relatives using BLUP. The use of BLUP is also widely studied in many other areas of research where the use of mixed linear models are relevant such as plant breeding ${ }^{19}$.

BLUP can be used in general linear mixed models that include both fixed and random effects. The simplest case is BLUP without pedigree, where genotypic effect is treated as an independent unobservable normally distributed random variable and no relationships between individuals are considered ${ }^{19}$. Compared with a model based on individual performance, pedigree based BLUP leads to more accurate predictions and result in larger genetic gain because it efficiently uses information from all relatives by constructing an additive genetic relationship matrix (A), under the circumstances where genetic relationships between relatives exists (Falconer and Mackay, 1996). The higher the additive genetic relationship between the genotype of interest with its relatives, the more information can be gained from records of these related genotypes ${ }^{19}$. With the rapid development of biochip technology, genomic BLUP (GBLUP) has been developed and widely applied because it is easy and straightforward to be implemented since technically it just needed the replacement of the $\mathbf{A}$ matrix in pedigreed based BLUP by a genomic matrix $(\mathbf{G})^{20}$. More recently, metabolomic BLUP (MBLUP) has been proposed by replacing the $\mathbf{A}$ or $\mathbf{G}$ matrix by a metabolomic similarity matrix $(\mathbf{M})$ and MBLUP has been shown as an promising method ${ }^{21}$.

In our previous study, around $36 \%$ of MFs were found having significant heritability and among which many were found to be correlated with MQ traits in spring barley ${ }^{13}$. With this information, it is worthwhile to investigate the role of MFs involved in the prediction of 
109 phenotypes for MQ traits. PLSR is a popular method used in the studies of metabolic profiles

$112^{22}$, and using metabolomic BLUP (MBLUP) model gave better prediction accuracies than the

111 BLUP model using genomic information for four of five quantitative traits investigated ${ }^{23}$.

112 The objectives of this study were to: 1) investigate the possibility of prediction of phenotypes

113 of malting quality traits using metabolomic information; 2) compare the ability of predictions

114 using PLSR and MBLUP models; 3) study the effect of different training population size on

115 the accuracy of prediction; 4) explore the possibility of metabolomic prediction within and

116 across location; and 5) compare different number of components considered in the PLSR model.

117

\section{Materials and methods}

119 All the data used are available in a public accessible repository ${ }^{24}$.

\section{Experiments}

121 In this study, a total of 2,667 plots of 564 spring barley malting lines were included. These

122 lines were part of the standard breeding program from Nordic Seed A/S. All experiments in 123 this study were conducted in land owned by Nordic Seed. There were no animal or human 124 experiments conducted for this research, the study also did not contain any GMO. Standard 125 farm operating procedure were used and therefore no ethical approval was needed for this study. 126 All the experiments involving plants adhered to plant ethics guidelines. Samples from two

127 locations in Denmark were used, and samples were taken from each plot individually and the 128 data covered three years from 2014 to 2016. In both locations, the fields were divided into trials, 129 which included 52 - 106 smaller plots $\left(8.25 \mathrm{~m}^{2}\right)$. Each trial was designed as a randomized 130 complete block comprising $20-45$ lines with three replicates of each line ${ }^{25}$. Each trial included 131 two control lines in three replications. As a consequence, testing was conducted in a number 132 of trials within each year-location combination. In total there were 139, 214 and 215 lines tested 133 in 2014, 2015, and 2016, respectively. 


\section{Measurements of malting quality traits}

135 The malt sample from each plot was milled and extracted in water in order to produce a wort

136 as described in the previous study ${ }^{6}$. The wort was used to measure five MQ traits which

137 included filtering speed (FS), extract yield (EY), wort color (WCO), beta glucan content (BG),

138 and wort viscosity (WV). The wort samples needed to be filtered first, and 20 minutes after

139 filtering begun, FS was scored by measuring the height of the liquid surface in the glass (cm

140 flow-through in $20 \mathrm{~min}$ ). EY was the percentage of dry matter in the filtered wort.

141 Spectrophotometer was used to determine WCO following the method of European Brewery

142 Convention (EBC) ${ }^{26}$. After the filtration, the wort samples were separated in two parts and all

143 wort phenotypes were obtained according to the Analytica-EBC 2004 manual. Briefly, one

144 sample of $25 \mathrm{ml}$ of wort was used for WV (mPa/s, Analytical-EBC 8.4) and EY (Analytical-

145 EBC 8.3). A second sample of 3 - $4 \mathrm{ml}$ of wort was used for BG (mg/L, Analytical-EBC 8.13.1)

146 and WCO (Analytical-EBC 8.5). Detailed description of MQ traits also can be found in a

147 previous study by Sarup, et al. ${ }^{6}$.

148 Metabolomic features and NMR intensities

149 The preparation of NMR analysis is described in detail in Guo, et al. ${ }^{13}$. MFs used in this study 150 were 24,018 NMR intensities which obtained from one-dimensional (1D) ${ }^{1} \mathrm{H}$ NMR spectra.

151 The NMR intensities were integrated over small chemical shift $(\delta)$ intervals and expressed in 152 parts per million (ppm) in the frequency range of $0.00 \mathrm{ppm}$ to $11.00 \mathrm{ppm}$. An in-house custom 153 Matlab script was used to process the spectra ${ }^{27}$. First an exponential apodization function 154 equivalent to $0.5 \mathrm{~Hz}$ line-broadening was used and then Fourier transformation was applied. 155 Afterwards, all spectra were referenced to the DSS-d6 signal, automatically phased, and 156 baseline corrected. After visual inspection data below $0.70 \mathrm{ppm}$ and above $9.00 \mathrm{ppm}$ was 157 removed as it did not contain any signal. The water peak which was in the range of $4.7 \mathrm{ppm}$ to $1584.9 \mathrm{ppm}$, and the region of the added standard which was $-0.2 \mathrm{ppm}$ to $0.2 \mathrm{ppm}$, were excluded. 
159 The raw data was then normalized using the probabilistic quotient method ${ }^{28}$, and the spectra

160 were aligned using icoshift ${ }^{29,30}$. Finally, the MFs were centered and standardized to a mean of

1610 and standard deviation as 1 in order to refine variation that could be attributed to experimental

162 sources and signal intensities ${ }^{12}$.

163 Statistical models and methods

164 Two models involved in the statistical analyses. The models were a metabolomic best linear 165 unbiased prediction (MBLUP) model and a partial least squares regression (PLSR) model.

\section{Metabolomic best linear unbiased prediction (MBLUP)}

167 MBLUP model was as follows:

$168 \boldsymbol{y}=\mathbf{1} \boldsymbol{\mu}+\boldsymbol{m}+\boldsymbol{e}$,

169 where $\boldsymbol{y}$ referred to the vector of each MQ trait, $\boldsymbol{\mu}$ was intercept, $\boldsymbol{m}$ was the vector metabolomic

170 effects, and $\boldsymbol{e}$ was a vector of residual terms that could not be explained by the other effects in

171 the model. In this model, $\boldsymbol{\mu}$ was a fixed parameter, $\boldsymbol{m}$ was a random parameter with

$172 \boldsymbol{m} \sim N\left(0, \boldsymbol{M} \sigma_{m}^{2}\right)$, and $\boldsymbol{e}$ was a random parameter with $\boldsymbol{e} \sim N\left(0, \boldsymbol{I} \sigma_{e}^{2}\right)$. The reason for only $\boldsymbol{\mu}$ taken

173 as fixed parameter, instead of following the model in our previous study to consider more fixed

174 parameters ${ }^{13}$, was because metabolomic information include environmental factors in addition

175 to genomic information. M denoted the metabolomic similarity matrix built from MFs using

176 the method as for building a genomic relationship matrix (G) computed using VanRaden

177 method 120 . Specifically, $\boldsymbol{M}=\frac{\boldsymbol{Q} \boldsymbol{Q}^{\prime}}{m}$, where $\mathbf{Q}$ is a $\mathrm{n} \times \mathrm{m}$ matrix of adjusted, centered and 178 scaled NMR intensities with $m=24,018$ (equal to number of MFs) and $n=2,667$ (equal to 179 number of samples). Both the build of $\mathbf{M}$ and the MBLUP analysis were carried out by using 180 the "qgg" R-package ${ }^{31}$.

181 The (co)variance components in the MBLUP model described in the previous section were 182 estimated by restricted maximum likelihood using “qgg” R-package ${ }^{31}$. 
183 The total variation of each MQ trait was calculated as the sum of variance components in

184 MBLUP model:

$185 \sigma_{P}^{2}=\bar{M} \sigma_{m}^{2}+\sigma_{e}^{2}$,

186 where $\bar{M}$ was the average diagonal of $\mathbf{M}$, which equal to 1 . The relative variance component

187 due to effects of $\boldsymbol{m}$ was $R V C_{m}=\frac{\bar{G} \sigma_{m}^{2}}{\sigma_{P}^{2}}$ which describe the proportion of total variation (across

188 fixed effects) in MQ traits that can be described by the MFs.

189 Partial least squares regression (PLSR)

190 The PLSR model decompose Q, the matrix of MFs, into orthogonal scores $\mathbf{T}$ and loadings $\mathbf{P}$ :

$191 Q=\boldsymbol{T P}$

192 so that regressing $\boldsymbol{y}$ not on $\mathbf{Q}$ itself but on the first $t$ columns of the scores $\boldsymbol{T}, t$ is the number of

193 components fitted in the model when using the package mentioned below. Detailed description of

194 PLSR method can be found in previous study on the "pls" package ${ }^{18}$, which was used in the

195 current study to carry out the PLS analysis.

\section{Cross-validation}

197 Three different leave-set-out (LSO) cross-validation strategies, in which the whole dataset was

198 divided into a training population (TP) and a validation population (VP), were investigated in

199 this study based on three different hypotheses regarding factors that influence prediction

200 accuracies. The first strategy, named SIZE, was to randomly leave out VP in order to create TP

201 of different size; the second strategy, named LINE, was to leave out VP according to line i.e.

202 all observations of a specific line; the third strategy, named LOC, was to leave out VP

203 according to location.

204 In the SIZE strategy, since the TP samples and VP samples were randomly selected, the TP

205 contained observations on the same lines, locations and years as in VP - although not the same

206 combinations of lines, locations and years. In the LINE strategy, one out of 564 lines was left

207 out so that the accuracy of predicting one line from all the other lines could be investigated, 
208 this strategy is similar to prediction of new lines. In the LOC strategy, one out of two locations

209 was left out, which means the accuracy of predicting one location based on data from the other

210 one location could be investigated.

211 For each strategy, cross-validation was carried out to evaluate the accuracy of metabolomic

212 prediction of five MQ traits using MFs. In order to study the effect of different size of TP in

213 SIZE strategy, eight scenarios were investigated in this study. These scenarios varied on TP

214 having the size of 50,100, 200, 500, 1,000, 1,500, 2,000, and 2,500. Since the selection of TP

215 was random, 15 replicates were carried out when selecting the TP. For the strategy of LINE,

216 data from 564 lines were left out separately so that 564 replicates were carried out in this

217 strategy. For the strategy of LOC, data from one of the two locations were left in turn so each

218 location were predicted based on the other location.

219 In each round of the cross-validation, according to the setup of TP size, a certain number of the

220 phenotypes were selected and then the rest of phenotypes were masked. The phenotypes of the

221 masked samples were predicted based on the TP together with the metabolomic information.

222 Thereafter, the correlation between phenotypes and the predicted values was calculated as the

223 accuracy of prediction. The accuracies obtained from strategy LINE and LOC were computed

224 based on both plot level and line mean level. This means, two accuracies were obtained for

225 each model in LINE and LOC strategies. In each round of the prediction in LINE and LOC

226 strategies, the predicted values for VP in this round were collected, and then when all the

227 rounds of prediction completed, predicted values were collected for the whole population.

228 Afterwards, the accuracy on plot level in LINE and LOC strategies was calculated as the 229 correlation between observed phenotypes and the predicted values of each plot, and the 230 accuracy of line mean was calculated as correlation between average observed phenotypes and

231 the average predicted values of each line. 
232 When applying PLSR model, compared with MBLUP model, a leave-one-sample-out (LOO)

233 cross-validation was carried out within the TP to train the model first, and afterwards the LSO

234 cross-validation was processed using the trained model from the preliminary LOO cross-

235 validation. In this study, different number of components considered in the PLSR model was

236 also compared, and the number of components were 5, 10, 20 and 50.

\section{Software and setup}

238 The cross-validation procedure using MBLUP was carried out by "qgg" package ${ }^{31}$ and the 239 procedure using PLSR model was carried out by "pls" package ${ }^{18}$. In all three strategies, both 240 MBLUP and PLSR models were applied, and the datasets utilized in each round of the cross241 validation were same for MBLUP and PLSR models to make sure they were comparable.

\section{Results}

244 In this study, the proportion of variance in malting quality (MQ) traits that can be explained by 245 effects of metabolomic features (MFs) was evaluated. Then the phenotype of MQ traits was predicted by using MFs through MBLUP and/or PLSR models. Different size of TP, and the different number of components considered in the PLSR models, prediction across line and location were also investigated.

\section{Descriptive statistics for malting quality traits}

250 Table 1 gives descriptive statistics of all the MQ traits analyzed in this study. There were 2,667 records analyzed for five MQ traits. The average of all the traits were 4.83 for FS, 82.66 for EY, 5.83 for $\mathrm{WCO}, 217.10$ for BG, and 1.47 for WV. The coefficient of phenotypic variance ranged from $2.21 \%$ for $\mathrm{EY}$ to $53.07 \%$ for $\mathrm{BG}$.

\section{Estimates of total variance of malting quality traits explained by metabolomic features}

255 A univariate MBLUP model was applied to estimate the proportion of the total variance including potential fixed effects in each MQ trait that can be explained by the MFs. The 
257 estimates were indicators for the proportion of total variance in MQ traits that is associated 258 with metabolites.

259 Figure 1 shows the estimated relative amount of total variance explained by MFs (RVCm) and 260 error in five MQ traits. The RVCm ranged from $0.926 \pm 0.011$ in FS to $0.981 \pm 0.002$ in BG.

261 For all the MQ traits, the effect of MFs explained very large proportions of the total variance.

262 RVCm in WCO, BG and WV were similar and larger than RVCm in FS and EY.

\section{Metabolomic prediction using MBLUP model}

264 A univariate MBLUP model was used for metabolomic prediction of the five MQ traits. The 265 cross-validation results from MBLUP model at each SIZE scenario are shown in Figure 2. As

266 shown in Figure 2, averaged across 15 replicates in the strategy of SIZE, the maximum 267 prediction accuracies using MBLUP model were $0.757 \pm 0.027$ for FS, $0.736 \pm 0.050$ for EY, $0.838 \pm 0.025$ for $\mathrm{WCO}, 0.780 \pm 0.033$ for $\mathrm{BG}$ and $0.816 \pm 0.028$ for $\mathrm{WV}$. In addition, for all traits, the maximum accuracy were obtained in the scenarios with 2,500 as TP size.

\section{Metabolomic prediction using PLSR model}

271 There were four PLSR models compared regarding the number of components utilized in the

272 model. The number of components considered were 5, 10, 20 and 50. As shown in Figure 3, averaged across 15 replicates, the maximum prediction accuracies using PLSR model were $0.741 \pm 0.026$ for $\mathrm{FS}, 0.730 \pm 0.053$ for $\mathrm{EY}, 0.826 \pm 0.024$ for $\mathrm{WCO}, 0.760 \pm 0.032$ for $\mathrm{BG}$ and $0.805 \pm 0.028$ for $\mathrm{WV}$. In addition, all the maximum accuracy were obtained when using PLSR model with 20 components, except FS, for which the maximum accuracy was provided by the PLSR model with 10 components, but it was very close to the accuracy provided by PLSR model with 20 components.

When the PLSR model considered 5 components, the prediction accuracy was low for all the MQ traits. With increase in the number of components considered in the PLSR model, the accuracy also generally increased. The accuracy kept increasing until the number of 
components reached 20. However, when the number of components increased further to 50, the accuracy decreased and in some cases even smaller than the accuracy from 5 components.

\section{Comparison of MBLUP and PLSR models}

285 The accuracy from MBLUP and the maximum accuracy among four PLSR models are plotted

286 for each SIZE scenario in Figure 2. The accuracy obtained from MBLUP model was smaller

287 than at the maximum accuracy from PLSR model when the TP size was small. When the TP size was 50, MBLUP yielded smaller accuracy in all the five MQ traits. With the increase of TP size, the accuracy from MBLUP increased rapidly and was larger than for the PLSR model. For example, in FS, MBLUP yielded higher accuracy than PLSR when the TP size just increased to 100. For all the traits, MBLUP yielded higher or same accuracy compared with all the PLSR models when the TP size reached 500.

\section{Metabolomic prediction with different training population size}

A total of eight sizes, including 50, 100, 200, 500, 1,000, 1,500, 2,000, and 2,500, of TP was compared. With the increase of TP size, the prediction accuracy increased regardless of the model used. When the TP size was small, PLSR could provide better predictions than MBLUP model, while along with the increase of TP size, the MBLUP outperformed PLSR models as soon as the data size reached 500 samples. As can be observed from Figure 2, though the general trend was higher accuracy obtained in the scenario of largest TP size. When the TP was increased beyond 1,000, the increase in accuracy was limited.

\section{Metabolomic prediction of new lines}

302 The second cross-validation strategy investigated in this study was LINE, in which the data from one line were masked as VP and the data from the other lines were treated as TP to predict the VP. This corresponds to predicting a new line based on metabolomic information only. 
30720 components generally yielded highest accuracy, only this PLSR model was conducted and

308 compared with MBLUP model in this strategy. As shown in Figure 4, when using MBLUP

309 model, the accuracy of plot ranged from $0.722 \pm 0.013$ for EY to $0.832 \pm 0.011$ for WCO, and

310 the accuracy of line mean ranged from $0.800 \pm 0.025$ for EY to $0.865 \pm 0.021$ for WV. The

311 MBLUP surpassed PLSR model though the difference are small.

312 Metabolomic prediction across location

313 The third cross-validation strategy investigated in this study was LOC, in which the data from

314 one location were masked as VP and the data from the other location were treated as TP to

315 predict the VP. There were two locations in the whole dataset, therefore, this strategy had two

316 rounds of prediction by treating each location as VP in each round. Same with LINE strategy,

317 both MBLUP and the PLSR with 20 components were carried out in this strategy. As shown

318 in Figure 5, the accuracy of plot ranged from. $0.517 \pm 0.017$ for EY to $0.684 \pm 0.014$ for FS,

319 the accuracy of line mean ranged from $0.713 \pm 0.030$ for WCO to $0.817 \pm 0.024$ for BG, when

320 using MBLUP model. The accuracy provided by PLSR model were similar with MBLUP.

\section{Discussion}

323 Metabolomic prediction using 24,018 metabolomic features (MFs) were carried for a total of

3242,667 plots of 564 spring barley malting lines each phenotyped for five malting quality (MQ)

325 traits. MBLUP and PLSR models were compared. Accuracy of cross-validation was 326 investigated by varying size of training population, also using leave-one-line-out and leave327 one-location-out strategies. In addition, considering number of components in the PLSR model 328 was also studied.

329 Descriptive statistics for malting quality traits

330 The descriptive statistics for MQ traits in the current study were similar with the previous study though the number of observations in the previous study was around three times larger than in 
332 the current study ${ }^{6}$. The standard deviation of most of the MQ traits in the current study were

333 smaller than the previous study and it is expected because in the previous study, 1,329 spring

334 malting barley lines were involved and the harvest was done in four different years and three

335 locations ${ }^{6}$, so that the samples from the current study was a subset of the previous study. The

336 more variation in the year and location compared with the current led to the larger variation in

337 phenotypes.

338 Estimates of total variance of malting quality traits explained by metabolomic features

339 The variance of five MQ traits explained by MFs was explored by using a univariate model

340 integrating a metabolomic similarity matrix and the proportion of metabolomic effects were

341 larger than $90 \%$ for all the five traits.

342 The utilization of metabolomic similarity matrix in the model aimed at dissection of the total

343 variance into a metabolomic part and a random error. The proportion of the variance of MFs

344 shows the extent that MFs can be used to predict total variance in MQ traits. In our previous

345 study, WCO, BG and WV were found to have significant phenotypic and genetic correlation

346 to a large proportion of the MFs ${ }^{13}$, similarly to a large extend, their total variance could be

347 explained by MFs. Potentially MQ traits of WCO, WV and BG can be predicted from the MFs

348 because MFs explains almost all the variance in these MQ traits. While among the two traits

349 (FS and EY) having relative lower proportion of correlation with MFs, they could not be

350 explained to the same very high degree by variation in metabolites.

351 The direct link between metabolites and phenotypic records in biological system provide the

352 potential of utilizing metabolomic features as an objective proxy for phenotype data ${ }^{32}$. The

353 fact that almost all the variation in MQ can be explained by the MFs confirmed that the MFs

354 could be used as the objective proxy for phenotype of interest and even more valuable and

355 meaningful when the phenotype of interest is difficult or expensive to be obtain.

356 Metabolomic prediction using MBLUP model 
357 The MBLUP model used for estimation of variance components was then used for metabolomic prediction of five MQ traits. The prediction accuracies using MBLUP model were quite promising as the maximum accuracy were all above 0.7 . This is higher than the

360 previous reported prediction accuracies for metabolomic prediction of plant phenotypes ${ }^{7,33-}$

$361{ }^{36}$. The higher prediction accuracy in this study is probably due to a larger number of unique 362 genotypes in the study and the fact that the NMR was performed directly on wort and not on e.g. leaves of the developing plant. When utilizing genomic information and fitting a genomic BLUP (GBLUP) model, the comparable accuracies for MQ traits were reported as from 0.28 to $0.68^{6}$. The accuracy of GBLUP in the previous study was lower than the accuracy of MBLUP in the current study can be due to metabolomic data included

367 information on both genetic factors as well as environmental factors. Thus the metabolomic information was closer related with phenotype observations than the genomic information. Though the spring barley lines involved in the current study were from the same breeding

370 program as the lines in the previous study ${ }^{6}$, the number of lines studied in the current study was a subset from the previous one, which can also lead to the difference in prediction accuracy. However the accuracy of GBLUP is expected to be even lower if using the same dataset as in the current study, which is smaller than and be a subset of the dataset in the previous study ${ }^{6}$.

One of the reasons for the high prediction accuracy using MBLUP could be because the total variance explained by MFs were large in all the five MQ traits. The phenotypes of MQ traits can be predicted very well and better than when using GBLUP, because most variation in MQ is expected to be reflected in the NMR spectra. The high accuracy also shows that there is no overfitting and MBLUP can explain and predict large variation in MQ. In addition, our previous study on the genetic and phenotypic correlation between MFs and MQ traits also showed a significant correlation between them, which can also be the reason for the high 
382 prediction accuracy using metabolomic information ${ }^{13}$. A subset of MFs were detected as 383 significantly heritable, and a further subset of these had significant genetic correlation with 384 MQ traits in our previous study ${ }^{13}$.Therefore, we carried out extra analysis in order to compare 385 the performance of MBLUP using different subsets of MFs. Three matrices were built 386 regarding to MFs included, the matrix using all the 24,018 MFs was M, the matrix using 387 significant heritable MFs was Ms, and the matrix using MFs which were significant heritable and also had significant genetic correlation with each trait was Mgs (varied across traits). The estimation of variance due to MFs were quite similar among the MBLUP models using M, Ms and Mgs. The accuracy of prediction for using these three MBLUP models with different training population size are shown in the Supplementary Figure S1. Very similar results obtained from three MBLUP models (M, Mgs, Ms) indicated that selecting the significant heritable MFs and/or MFs having significant genetic correlation with traits did not improve the prediction accuracy of MBLUP. When applying MBLUP in the breeding system, breeders can directly utilize all the MFs instead of filtering out some part of MFs which may involve more work, cost, and potential for errors.

397 The performance of GBLUP and MBLUP was investigated in Drosophila, where the prediction accuracy for two behavioral traits was below 0.1 when based on GBLUP and then increased to above 0.4 when using MBLUP. Such an increase have also been found for two environmental stress resistance traits in Drosophila ${ }^{23}$. In the plant field, metabolic information was introduced 401 into prediction of complex traits by Riedelsheimer, et al. ${ }^{33}$, where the authors presented a 402 complementary approach to exploit large-scale genomic and metabolic information in hybrid testcrosses. The MBLUP was also investigated in a previous study ${ }^{37}$, where metabolomics data were used to predict the performance agronomic traits in wheat, and metabolomic information were found as providing strong predictive power for number of grains per spike and plant 406 height ${ }^{37}$. 


\section{Metabolomic prediction using PLSR model}

408 Four PLSR models were compared with different the number of components considered in the

409 model. The maximum accuracies provided by PLSR models were smaller than for the MBLUP

410 model. Increasing the number of components considered in the PLSR models generally led to

411 the increase of prediction accuracy while when the number of components reached 50, the

412 prediction accuracy was not larger than the one provided by the models only considered 20

413 components. These results indicated that the non-linear relationship between the number of

414 components and the performance of the prediction. For all the MQ traits investigated in the

415 current study, 20 components were already enough to provide good prediction accuracy though

416 the exact number of components varied a bit from trait to trait. In a study of genomic selection

417 for pork $\mathrm{pH}$ traits, 30 was found as the optimal number of components considered in the PLSR

418 analysis ${ }^{38}$.

419 PLSR has also been suggested as an efficient method to analyze genomic data, because of its 420 ability to handle large data sets and its prediction ability, and the PLSR approach is particularly 421 suitable to predict dependent variables from a very large number of predictors and especially 422 the predictors might be highly correlated with each other ${ }^{39}$. The accuracy of prediction for 423 yield traits in French dairy cattle were similar between PLSR and GBLUP models but in no 424 case PLSR provided higher accuracy than GBLUP ${ }^{39}$. It was also reported that an increase in 425 the number of relevant variables and observations contributed to the improvement in the 426 precision of the model parameters, which was one desirable property of PLSR model ${ }^{16}$.

\section{Comparison of MBLUP and PLSR models}

428 The comparison of MBLUP and PLSR models showed that MBLUP generally outperformed 429 PLSR for all the traits, when the TP size larger than 500. PLSR could be a better choice than 430 MBLUP only when the TP was small. In a previous study the $\mathrm{Xu}$, et al. ${ }^{21}$ analyzed a hybrid 431 population of rice, and showed that the MBLUP model was superior to PLSR model ${ }^{21}$. A 
432 similar situation was also found when utilizing genomic information instead of metabolomic

433 information. For example, a study on rice also investigated the GBLUP model and PLSR using

434 genomic information, showed that the GBLUP outperformed PLSR ${ }^{21}$. The superiority of

435 BLUP model was also found in the study on genomic prediction in French Holstein and

436 Montbéliarde breeds ${ }^{40}$. In addition to the better performance of MBLUP, it is also easy to

437 implement, needs low demands regarding computation power, time and skill for the breeder,

438 which makes MBLUP is more attractive in the practical breeding.

\section{Metabolomic prediction with different training population size}

440 A total of eight TP sizes from 50 to 2,500 were compared in this study. The results showed that

441 the prediction accuracy generally increased with increasing TP size. Though the accuracy

442 increased all the way from smallest TP until the largest dataset, the increase in accuracy was

443 much smaller when the TP were larger than 1,000. The impact of TP size on the prediction

444 accuracy had been demonstrated in the genomic prediction while rare in the metabolomic

445 prediction using metabolomic information ${ }^{41,42}$. For example, the accuracy of genomic

446 prediction in wheat has been investigated regarding to different population sizes and the results

447 indicated that TP of around 700 lines were enough to yield the highest prediction accuracy. ${ }^{43}$.

448 Metabolomic prediction across line/location

449 In addition to the first cross-validation strategy which selecting TP randomly within the whole 450 population, two more strategies were investigated either predict the VP from different lines or 451 growing in different locations. The accuracy of predicting plot MQ from these two strategies 452 were smaller than when the TP randomly selected from the whole population. The reason is 453 because when selecting TP from the whole population randomly, the observations on the same 454 lines and/or locations were involved in TP and VP, which increased the degree of the 455 metabolomic similarity between TP and VP. 
456 In this study, 564 lines were harvested in three years separately, which means there was almost

457 no lines involved in two or three years. This design created difficulty in the metabolomic

458 prediction across year based on the current dataset. The across year metabolomic prediction

459 could be better investigated when a dataset including overlap of lines been planted in different

460 years is available.

461

462 Conclusion

463 Records of five malting quality (MQ) traits and metabolomic features (MFs) for 2,667 plots of 464564 spring malting barley lines that were grown in two locations were studied. The ability of 465 prediction based on metabolomic information was investigated.

466 The proportion of variance in MQ traits that can be explained by effects of MFs was above 0.9

467 for all traits when using all the records. The phenotype of MQ traits could be predicted by MFs 468 through MBLUP and/or PLSR models. The prediction accuracy when using MBLUP was 469 larger than 0.7 and generally surpassed PLSR models when size of training population (TP) 470 larger than 500. When the size of TP smaller than 500, PLSR provided better accuracy than 471 MBLUP. The prediction accuracy increased along with increasing TP size but when the 472 population size reached 1,000 , the rate of increase was very small. The number of components 473 considered in the PLSR models can affect the performance of PLSR models and 20 was the optimal number. In addition, the prediction accuracy was also explored regarding to using the TP to predict the validation population (VP) in a different year or location. The results showed 476 that it was possible carry out the prediction across line/location with the accuracy of plot ranged 477 from 0.5 to 0.8 , and the accuracy of line mean ranged from 0.7 to 0.9 .

478 In conclusion, it is possible to carry out prediction for phenotypes of malting quality traits using 479 metabolomic information. MBLUP is an ideal model for the prediction when TP size larger 
Metabolomic prediction of barley malting quality

480 than 500. The results from the current study indicate that barley breeders can predict MQ based

481 on MFs from the wort and have significant implications for the practical barley breeding. 


\section{References}

4831 Gupta, M., Abu-Ghannam, N. \& Gallaghar, E. Barley for Brewing: Characteristic 484 Changes during Malting, Brewing and Applications of its By-Products. Comprehensive 485 Reviews in Food Science and Food Safety 9, 318-328, 486 doi:https://doi.org/10.1111/j.1541-4337.2010.00112.x (2010).

4872 Burger, W. C. \& LaBerge, D. E. Malting and Brewing Quality. Barley, 367-401, 488 doi:https://doi.org/10.2134/agronmonogr26.c13 (1985).

4893 MacLeod, L. \& Evans, E. Malting. Reference Module in Food Science, 490 doi:https://doi.org/10.1016/B978-0-08-100596-5.00153-0 (2016).

4914 Li, C. D., Cakir, M. \& Lance, R. in Genetics and Improvement of Barley Malt Quality 492 (eds Guoping Zhang \& Chengdao Li) 260-292 (Springer Berlin Heidelberg, 2010).

4935 Gao, W. et al. Fine mapping of a malting-quality QTL complex near the chromosome 494 4H S telomere in barley. Theor Appl Genet 109, 750-760, doi:10.1007/s00122-004$495 \quad 1688-7(2004)$.

4966 Sarup, P. et al. Genomic prediction for malting quality traits in practical barley breeding 497 programs. bioRxiv, 2020.2007.2030.228007, doi:10.1101/2020.07.30.228007 (2020).

4987 Meyer, R. C. et al. The metabolic signature related to high plant growth rate in 499 Arabidopsis thaliana. P Natl Acad Sci USA 104, 4759-4764, $500 \quad$ doi:10.1073/pnas.0609709104 (2007).

5018 Jewett, M. C., Hofmann, G. \& Nielsen, J. Fungal metabolite analysis in genomics and 502 phenomics. Current Opinion in Biotechnology 17, 191-197, 503 doi:https://doi.org/10.1016/j.copbio.2006.02.001 (2006).

5049 Saito, K. \& Matsuda, F. Metabolomics for Functional Genomics, Systems Biology, and 505 Biotechnology. Annual Review of Plant Biology 61, 463-489, doi:10.1146/annurev.arplant.043008.092035 (2010). 
50710 Roessner, U. \& Bowne, J. What is metabolomics all about? BioTechniques 46, 363-365, $508 \quad$ doi:10.2144/000113133 (2009).

50911 Lu, W. et al. Metabolite Measurement: Pitfalls to Avoid and Practices to Follow. Annu $510 \quad$ Rev Biochem 86, 277-304, doi:10.1146/annurev-biochem-061516-044952 (2017).

$511 \quad 12$ Aliakbari, A. et al. Genetic variance of metabolomic features and their relationship with 512 body weight and body weight gain in Holstein cattle1. Journal of Animal Science 97, 513 3832-3844, doi:10.1093/jas/skz228 (2019).

51413 Guo, X. et al. Genetic Variance of Metabolomic Features and Their Relationship With 515 Malting Quality Traits in Spring Barley. Frontiers in Plant Science 11, 516 doi:10.3389/fpls.2020.575467 (2020).

51714 Wold, H. Soft Modelling by Latent Variables: The Non-Linear Iterative Partial Least 518 Squares (NIPALS) Approach. Journal of Applied Probability 12, 117-142, 519 doi:10.1017/S0021900200047604 (1975).

$52015 \mathrm{Xu}, \mathrm{S} . \& \mathrm{Hu}, \mathrm{Z}$. Methods of plant breeding in the genome era. Genetics Research 92 , $521 \quad$ 423-441, doi:10.1017/S0016672310000583 (2010).

52216 Wold, S., Sjöström, M. \& Eriksson, L. PLS-regression: a basic tool of chemometrics. 523 Chemometrics and Intelligent Laboratory Systems 58, 109-130, 524 doi:https://doi.org/10.1016/S0169-7439(01)00155-1 (2001).

52517 Carrascal, L., Galván, I. \& Gordo, O. Partial least squares regression as an alternative 526 to current regression methods used in ecology. Oikos 118, 681-690, 527 doi:10.1111/j.1600-0706.2008.16881.x (2009).

52818 Mevik, B.-H. \& Wehrens, R. The pls Package: Principal Component and Partial Least 529 Squares Regression in R. 2007 18, 23, doi:10.18637/jss.v018.i02 (2007). 
53019 Piepho, H. P., Möhring, J., Melchinger, A. E. \& Büchse, A. BLUP for phenotypic 531 selection in plant breeding and variety testing. Euphytica 161, 209-228, 532 doi:10.1007/s10681-007-9449-8 (2008).

53320 VanRaden, P. M. Efficient methods to compute genomic predictions. Journal of dairy $534 \quad$ science 91, 4414-4423, doi:10.3168/jds.2007-0980 (2008).

53521 Xu, S., Xu, Y., Gong, L. \& Zhang, Q. Metabolomic prediction of yield in hybrid rice. The Plant Journal 88, 219-227, doi:https://doi.org/10.1111/tpj.13242 (2016).

53722 Sarup, P., Pedersen, S. M. M., Nielsen, N. C., Malmendal, A. \& Loeschcke, V. The 538 Metabolic Profile of Long-Lived Drosophila melanogaster. PLOS ONE 7, e47461, 539 doi:10.1371/journal.pone.0047461 (2012).

54023 Rohde, P. D., Kristensen, T. N., Sarup, P., Muñoz, J. \& Malmendal, A. Prediction of complex phenotypes using the \&lt;em\&gt;Drosophila\&lt;/em\&gt; metabolome. bioRxiv, 2020.2006.2011.145623, doi:10.1101/2020.06.11.145623 (2020).

54324 Guo, X. (Mendeley Data, 2020).

54425 Nielsen, N. H. et al. Genomic Prediction of Seed Quality Traits Using Advanced Barley Breeding Lines. PloS one 11, e0164494-e0164494, doi:10.1371/journal.pone.0164494 (2016).

54726 Bishop, L. R. EUROPEAN BREWERY CONVENTION TESTS OF THE E.B.C. COLOUR DISCS FOR WORT AND BEER. Journal of the Institute of Brewing 72, 443-451, doi:10.1002/j.2050-0416.1966.tb02988.x (1966).

55027 Haggart, G., Pearce, J. \& Sands, C. ghaggart, 〈https://zenodo.org/record/3077413> (2019).

55228 Dieterle, F., Ross, A., Schlotterbeck, G. \& Senn, H. Probabilistic quotient normalization as robust method to account for dilution of complex biological mixtures. Application in 1H NMR metabonomics. Anal. Chem. 78, 4281-4290 (2006). 
55529 Savorani, F., Tomasi, G. \& Engelsen, S. B. icoshift: A versatile tool for the rapid 556 alignment of 1D NMR spectra. J Magn Reson 202, 190-202, 557 doi:10.1016/j.jmr.2009.11.012(2010).

$55830 \quad \mathrm{Vu}, \mathrm{T}$. N. \& Laukens, K. Getting your peaks in line: a review of alignment methods for 559 NMR spectral data. Metabolites 3, 259-276 (2013).

$56031 \quad$ Rohde, P. D., Fourie Sørensen, I. \& Sørensen, P. qgg: an R package for large-scale quantitative genetic analyses. Bioinformatics 36, 2614-2615, doi:10.1093/bioinformatics/btz955 (2019).

56332 Daygon, V. \& Fitzgerald, M. Application of metabolomics for providing a new generation of selection tools for crop improvement. Hot Topics in Metabolomics: Food and Nutrition, 106, doi:10.4155/9781909453821 (2013).

56633 Riedelsheimer, C. et al. Genomic and metabolic prediction of complex heterotic traits in hybrid maize. Nature Genetics 44, 217-220, doi:10.1038/ng.1033 (2012).

56834 Gärtner, T. et al. Improved heterosis prediction by combining information on DNAand metabolic markers. PLoS One 4, e5220, doi:10.1371/journal.pone.0005220 (2009).

57035 Steinfath, M. et al. Discovering plant metabolic biomarkers for phenotype prediction using an untargeted approach. Plant Biotechnol J 8, 900-911, doi:10.1111/j.14677652.2010.00516.x (2010).

$573 \quad 36 \quad$ Feher, K. et al. Deducing hybrid performance from parental metabolic profiles of young primary roots of maize by using a multivariate diallel approach. PLoS One 9, e85435, doi:10.1371/journal.pone.0085435 (2014).

57637 Shi, T. et al. Metabolomics analysis and metabolite-agronomic trait associations using kernels of wheat (Triticum aestivum) recombinant inbred lines. The Plant Journal 103, 279-292, doi:https://doi.org/10.1111/tpj.14727 (2020). 
57938 Silveira, F. G. d. et al. The optimal number of partial least squares components in $580 \quad$ genomic selection for pork pH. Cienc Rural 47 (2017).

58139 Colombani, C. et al. A comparison of partial least squares (PLS) and sparse PLS 582 regressions in genomic selection in French dairy cattle. Journal of dairy science $\mathbf{9 5}$, 583 2120-2131, doi:https://doi.org/10.3168/jds.2011-4647 (2012).

58440 Colombani, C. et al. Application of Bayesian least absolute shrinkage and selection operator (LASSO) and BayesC $\pi$ methods for genomic selection in French Holstein and Montbéliarde breeds. Journal of dairy science 96, 575-591, doi:https://doi.org/10.3168/jds.2011-5225 (2013).

58841 Goddard, M. Genomic selection: prediction of accuracy and maximisation of long term response. Genetica 136, 245-257, doi:10.1007/s10709-008-9308-0 (2009).

$59042 \quad$ Daetwyler, H. D., Villanueva, B. \& Woolliams, J. A. Accuracy of predicting the genetic risk of disease using a genome-wide approach. PLoS One 3, e3395, doi:10.1371/journal.pone.0003395 (2008).

59343 Cericola, F. et al. Optimizing Training Population Size and Genotyping Strategy for 594 Genomic Prediction Using Association Study Results and Pedigree Information. A Case of Study in Advanced Wheat Breeding Lines. PloS one 12, e0169606-e0169606, doi:10.1371/journal.pone.0169606 (2017). 


\section{Conflict of Interest}

599 The authors declare that the research was conducted in the absence of any commercial or 600 financial relationships that could be construed as a potential conflict of interest. PS, AJ are

601 employed by Nordic Seed A/S. XG is employed by both Aarhus University and SEGES. The

602 funders had no influence on the study design or choice of analysis methods.

\section{Author Contributions}

604 XG implemented and carried out the statistical analysis, interpreted the results and drafted the

605 manuscript. AJ, PS, JJ and XG contributed to the experimental design and PS, JJ and XG

606 developed the statistical models. All authors participated in interpreting the results and all 607 authors read and approved the final manuscript.

\section{Funding}

609 This project was funded by the Innovation Fund Denmark (Grant number: 5184-00032B) and

610 by Green Development and Demonstration Programme (GUDP, Grant number: 34009-19-

611 1586).

\section{Acknowledgments}

613 The authors are grateful to Vahid Edriss, Nanna Hellum Kristensen, Jens Due Jensen, and Jette

614 Andersen from Nordic Seed for field and laboratory work, Frans A. A. Mulder, Lars Alf Jensen

615 and Benjamin Wahlqvist from Aarhus University for sample preparation and performing NMR

616 measurements, and Palle Duun Rohde from Aalborg University for very helpful discussion.

\section{Abbreviations}

618 BG: beta glucan

619 BLUP: best linear unbiased prediction

620 BV: breeding value

$621 \mathrm{CV}$ : coefficient of phenotypic variance 
622 GBLUP: genomic best linear unbiased prediction

623 G: genomic relationship matrix

624 EBC: European Brewery Convention

625 EBV: expected breeding value

626 EY: extract yield

627 FS: filtering speed

628 LOO: leave-one-sample-out

629 LSO: leave-set-out

630 M: metabolomic similarity matrix built from all metabolomic features

631 MBLUP: metabolomic best linear unbiased prediction

632 MFs: metabolomic features

633 Mgs: metabolomic similarity matrix built from significant heritable metabolomic features

634 having significant genetic correlation with metabolomic traits

635 MQ: malting quality

636 Ms: metabolomic similarity matrix built from significant heritable metabolomic features

637 NMR: nuclear magnetic resonance

638 PLS: partial least squares

639 PLSR: Partial least squares regression

640 ppm: parts per million

641 RVC: relative variance component

642 TP: training population

643 VP: validation population

644 WCO: wort color

645 WV: wort viscosity 


\section{Tables}

647 Table 1. Descriptive statistics for malting quality traits.

\begin{tabular}{|c|c|c|c|c|c|c|c|}
\hline Trait & $\begin{array}{c}\text { No. of } \\
\text { records }\end{array}$ & Unit & Average & S.D. & Min & Max & CV \\
\hline FS & 2,667 & $\begin{array}{c}\mathrm{cm} / 2 \\
0 \mathrm{~min}\end{array}$ & 4.83 & 0.61 & 2.30 & 6.30 & $12.72 \%$ \\
\hline EY & 2,667 & $\%$ & 82.66 & 1.82 & 70.38 & 92.39 & $2.21 \%$ \\
\hline $\mathbf{W C O}$ & 2,667 & $\begin{array}{c}\mathrm{EBC} \\
\text { units }\end{array}$ & 5.83 & 0.83 & 3.59 & 8.99 & $14.23 \%$ \\
\hline $\mathbf{B G}$ & 2,667 & $\mathrm{mg} / \mathrm{L}$ & 217.10 & 115.23 & 70.00 & 751.19 & $53.07 \%$ \\
\hline $\mathbf{W V}$ & 2,667 & $\begin{array}{c}\mathrm{mPa} \\
\mathrm{s}\end{array}$ & 1.47 & 0.06 & 1.29 & 1.73 & $4.27 \%$ \\
\hline
\end{tabular}

648 Trait: $\mathrm{FS}=$ filtering speed, $\mathrm{EY}=$ extract yield, $\mathrm{WCO}=$ wort color, $\mathrm{BG}=$ beta glucan, $\mathrm{WV}=$ wort viscosity; $649 \mathrm{CV}$ is phenotypic coefficient of variance. 


\section{$650 \quad$ Figures}

651 Figure 1 Proportion of total variance explained by metabolomic features and error in malting

652 quality traits

653 Trait: $\mathrm{FS}=$ filtering speed, $\mathrm{EY}=$ extract yield, $\mathrm{WCO}=$ wort color, $\mathrm{BG}=$ beta glucan, $\mathrm{WV}=$ wort viscosity; $\mathrm{y}$ -

654 axis is relative variance component; $m$ is relative variance of metabolomic effects and $\mathrm{e}$ is relative variance of 655 residuals. 
657 Figure 2 Accuracy of prediction for malting quality traits using MBLUP and PLSR models

658 with different training population size

659 Trait: FS = filtering speed, EY = extract yield, $\mathrm{WCO}=$ wort color, $\mathrm{BG}=$ beta glucan, $\mathrm{WV}=$ wort viscosity; $\mathrm{x}-$

660 axis is training population size, y-axis is accuracy of prediction which is the correlation between observed and

661 predicted phenotypes; MBLUP is metabolomic best linear unbiased prediction model, PLSR is partial least

662 squares regression model; PLSR at each point are the results from PLSR model with best number of components. 
665 Figure 3 Accuracy of prediction for malting quality traits using PLSR models with different 666 training population size

667 Trait: FS = filtering speed, $\mathrm{EY}=$ extract yield, $\mathrm{WCO}=$ wort color, $\mathrm{BG}=$ beta glucan, $\mathrm{WV}=$ wort viscosity; $\mathrm{x}$ 668 axis is training population size, $y$-axis is accuracy of prediction which is the correlation between observed and 669 predicted phenotypes; PLSR is partial least squares regression model; PLSR_05 - PLSR_50 are partial least 670 squares regression models with different number of components $(5,10,20,50)$. 
672

673 Figure 4 Accuracy of prediction for malting quality traits across line using MBLUP and PLSR 674 models

675 Trait: FS = filtering speed, $\mathrm{EY}=$ extract yield, $\mathrm{WCO}=$ wort color, $\mathrm{BG}=$ beta glucan, $\mathrm{WV}=$ wort viscosity; $\mathrm{y}-$ 676 axis is accuracy of prediction which is the correlation between observed and predicted phenotypes; MBLUP is metabolomic best linear unbiased prediction model; PLSR is partial least squares regression model with 20 components; plot is accuracy of plot, mean is accuracy of line mean. 
682 Figure 5 Accuracy of prediction for malting quality traits across location using MBLUP and 683 PLSR models

684 Trait: FS = filtering speed, EY = extract yield, $\mathrm{WCO}=$ wort color, $\mathrm{BG}=$ beta glucan, $\mathrm{WV}=$ wort viscosity; $\mathrm{y}$ 685 axis is accuracy of prediction which is the correlation between observed and predicted phenotypes; MBLUP is 686 metabolomic best linear unbiased prediction model; PLSR is partial least squares regression model with 20 687 688 components; plot is accuracy of plot, mean is accuracy of line mean. 


\section{Supplementary Material}

690

691

692

693

694

695

696

697
Figure S1 Accuracy of prediction for malting quality traits using MBLUP models with different sets of metabolomic features

Trait: FS = filtering speed, $\mathrm{EY}=$ extract yield, $\mathrm{WCO}=$ wort color, $\mathrm{BG}=$ beta glucan, $\mathrm{WV}=$ wort viscosity; $\mathrm{x}-$ axis is training population size, y-axis is accuracy of prediction which is the correlation between observed and predicted phenotypes; MBLUP is metabolomic best linear unbiased prediction model, M is MBLUP using all metabolomic features, Mgs is MBLUP using metabolomic features having significant genetic correlation with each trait and significantly heritable, Ms is MBLUP using metabolomic features significant heritable. 
Figures
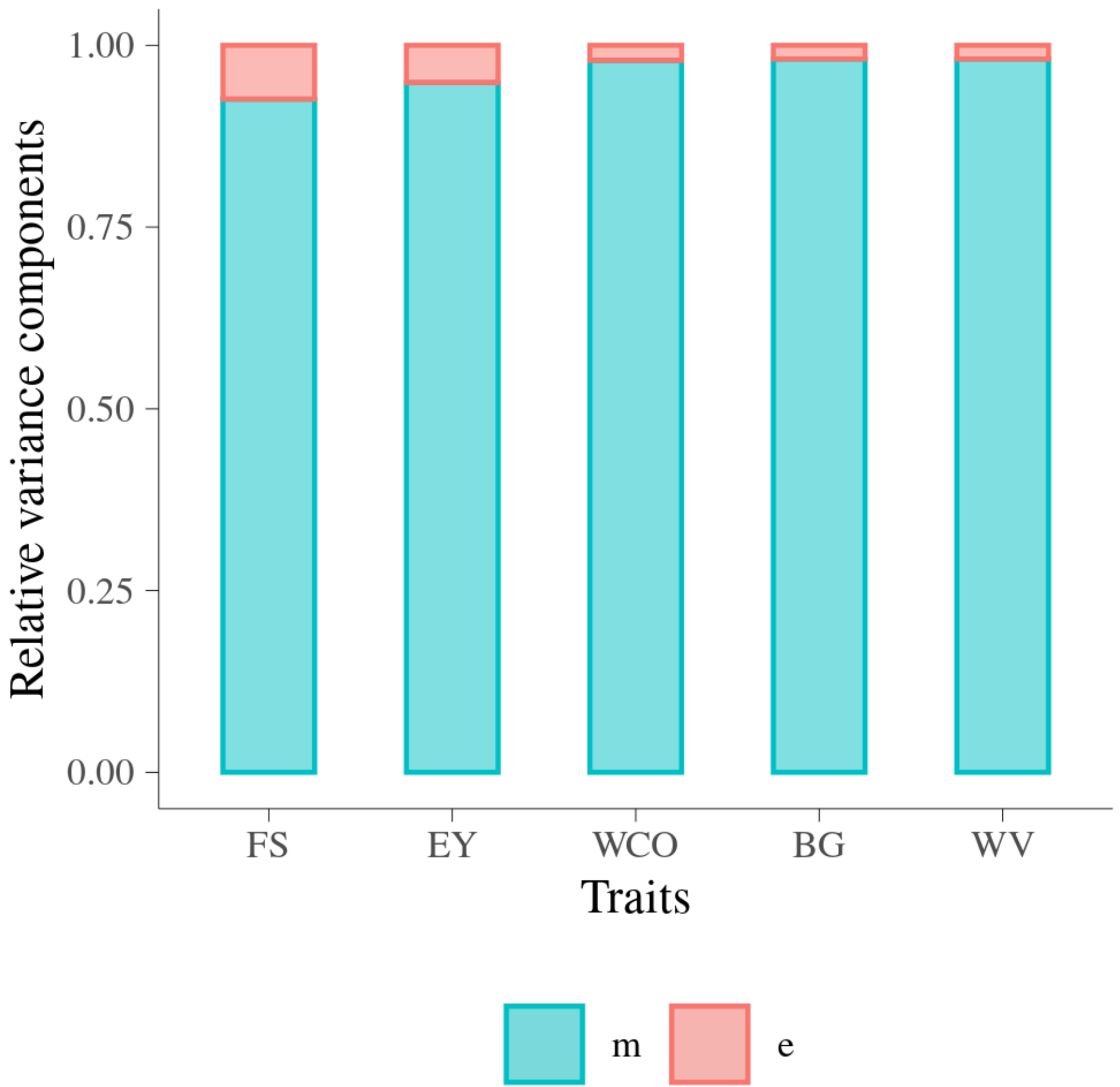

\section{Figure 1}

Proportion of total variance explained by metabolomic features and error in malting quality traits Trait: FS = filtering speed, $\mathrm{EY}=$ extract yield, $\mathrm{WCO}=$ wort color, $\mathrm{BG}$ = beta glucan, $\mathrm{WV}=$ wort viscosity; $\mathrm{y}$-axis is relative variance component; $m$ is relative variance of metabolomic effects and $e$ is relative variance of residuals. 


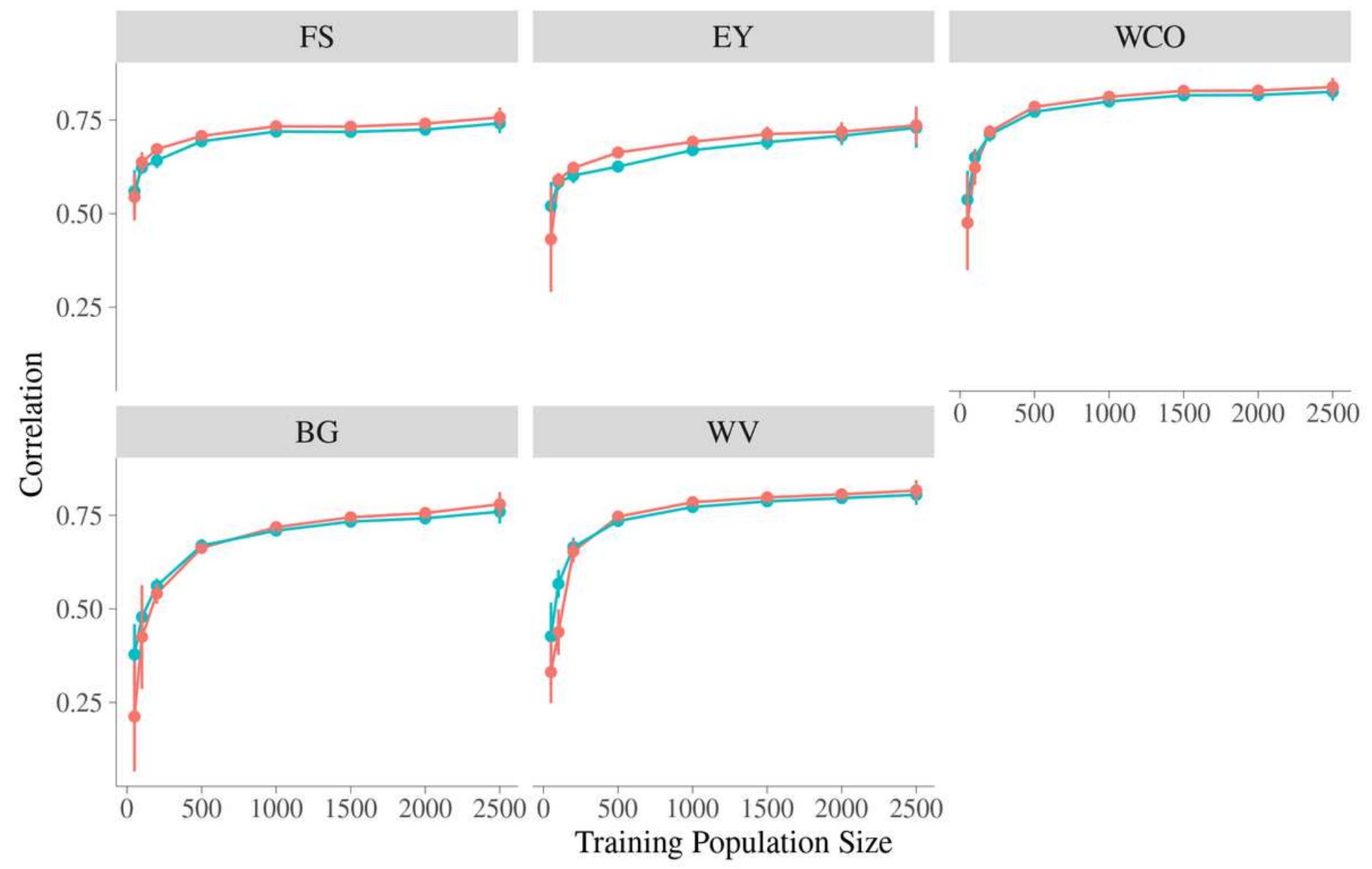

Figure 2

Accuracy of prediction for malting quality traits using MBLUP and PLSR models with different training population size Trait: FS = filtering speed, $E Y=$ extract yield, $W C O=$ wort color, $B G=$ beta glucan, $W V=$ wort viscosity; $x$-axis is training population size, $y$-axis is accuracy of prediction which is the correlation between observed and predicted phenotypes; MBLUP is metabolomic best linear unbiased prediction model, PLSR is partial least squares regression model; PLSR at each point are the results from PLSR model with best number of components. 


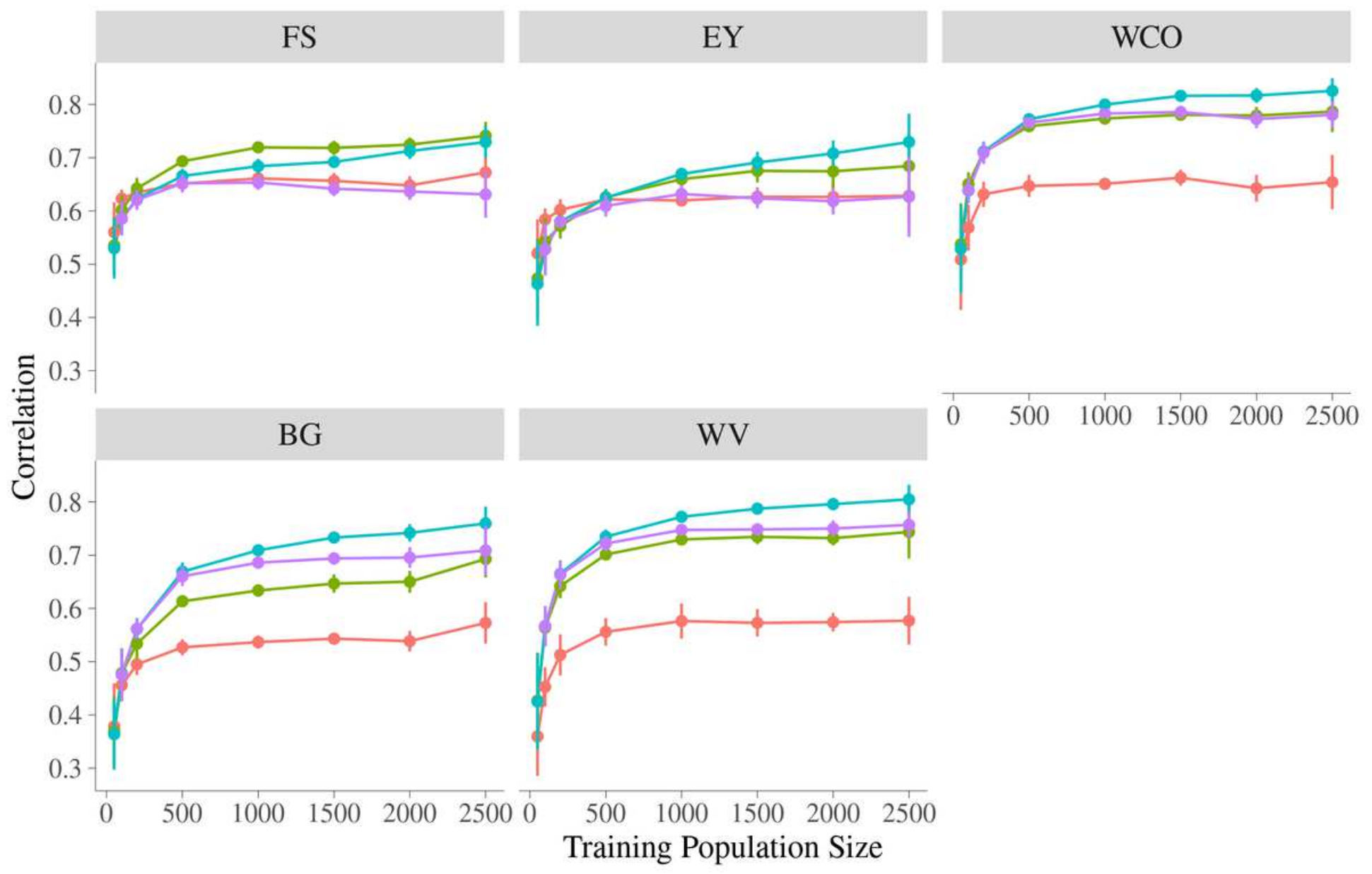

PLSR_05 $\rightarrow$ PLSR_10 $\rightarrow$ PLSR_20 $\rightarrow$ PLSR_50

\section{Figure 3}

Accuracy of prediction for malting quality traits using PLSR models with different training population size Trait: FS = filtering speed, $E Y=$ extract yield, $W C O=$ wort color, $B G=$ beta glucan, $W V$ = wort viscosity; $x-$ axis is training population size, $y$-axis is accuracy of prediction which is the correlation between observed and predicted phenotypes; PLSR is partial least squares regression model; PLSR_05 - PLSR_50 are partial least squares regression models with different number of components $(5,10,20,50)$. 


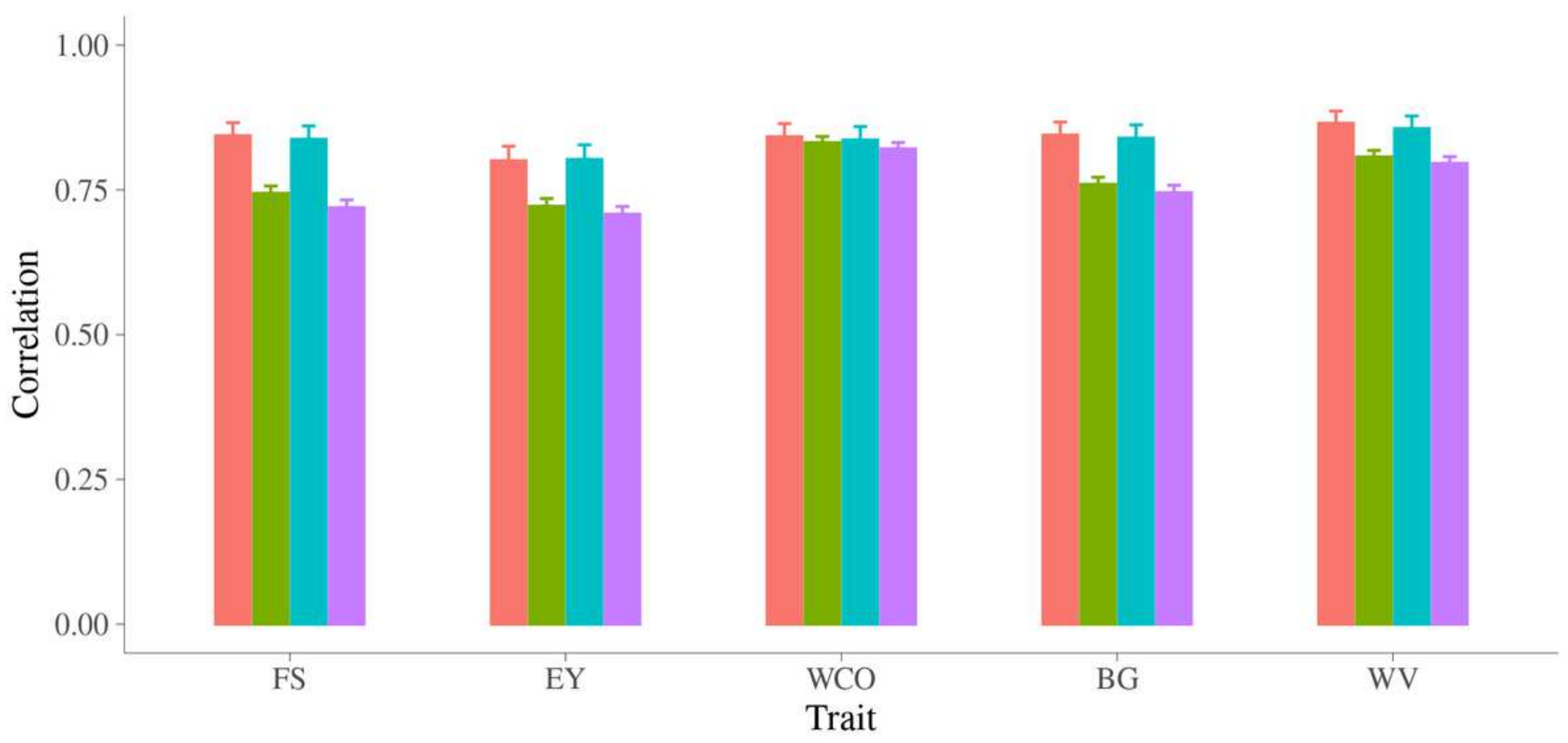

MBLUP_mean $\quad$ MBLUP_plot $\quad$ PLSR_mean $\quad$ PLSR_plot

\section{Figure 4}

Accuracy of prediction for malting quality traits across line using MBLUP and PLSR models Trait: FS = filtering speed, $E Y=$ extract yield, $W C O=$ wort color, $B G$ = beta glucan, $W V=$ wort viscosity; $y$-axis is accuracy of prediction which is the correlation between observed and predicted phenotypes; MBLUP is metabolomic best linear unbiased prediction model; PLSR is partial least squares regression model with 20 components; plot is accuracy of plot, mean is accuracy of line mean. 


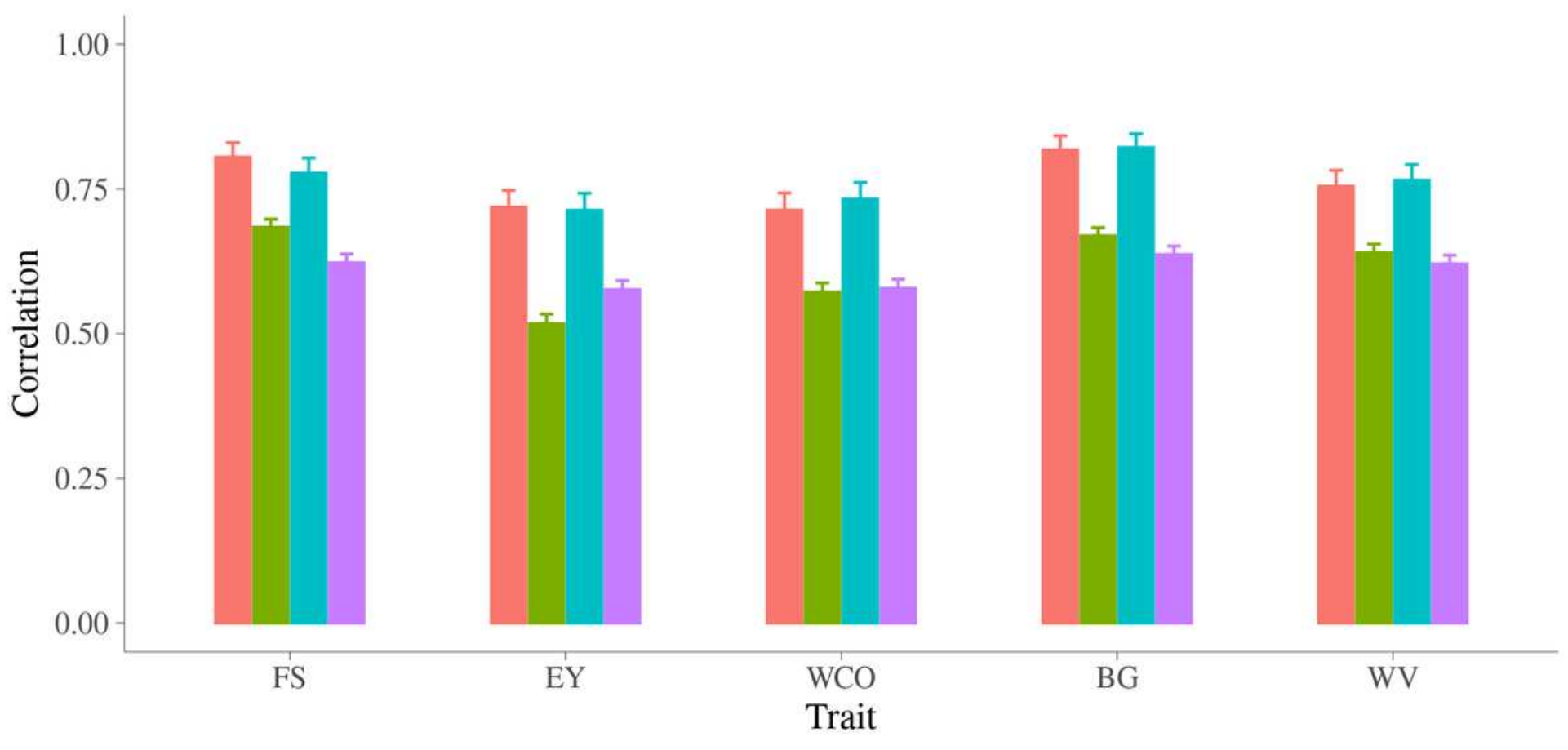

MBLUP_mean $\quad$ MBLUP_plot $\quad$ PLSR_mean $\quad$ PLSR_plot

\section{Figure 5}

Accuracy of prediction for malting quality traits across location using MBLUP and PLSR models Trait: FS = filtering speed, $\mathrm{EY}=$ extract yield, $\mathrm{WCO}=$ wort color, $\mathrm{BG}$ = beta glucan, $\mathrm{WV}=$ wort viscosity; $y$-axis is accuracy of prediction which is the correlation between observed and predicted phenotypes; MBLUP is metabolomic best linear unbiased prediction model; PLSR is partial least squares regression model with 20 components; plot is accuracy of plot, mean is accuracy of line mean.

\section{Supplementary Files}

This is a list of supplementary files associated with this preprint. Click to download.

- phenometaxiangyusup.docx 